\title{
29. HYDROGEN IN THE REGIONS OF FOUR STELLAR CLUSTERS
}

\author{
H. M. TOVMASSIAN
}

Byurakan Astrophysical Observatory, Erevan, Armenia, U.S.S.R.

Young clusters of $O$ type and the corresponding stellar associations, together with the associated hydrogen complexes, make up the spiral arms of our Galaxy. Thus the investigation of the distribution of hydrogen and its relation to the clusters is of definite interest.

The present report gives preliminary results of observations of the hydrogen line in the regions of four clusters, aiming to reveal the gas clouds associated with these clusters.

The observations were made in 1965 with the 210-feet radio telescope of the Australian National Radio Astronomical Observatory at Parkes. Most of the observations were made with the 48-channel receiver with bandwidths of the channels of $33 \mathrm{kHz}$, and some of them with the 15-channel narrow-band receiver with bandwidths of the channels of $9 \mathrm{kHz}$. The noise level has been kept almost the same during both observations by increasing the integration time with the narrow-band receiver. Peakto-peak fluctuations with both receivers were about $1.5 \mathrm{~K}$.

The observed area in each case exceeds that of the corresponding clusters by several hundred times. Most of the scans have been made along the galactic plane through the positions of the clusters or shifted by $6^{\prime}, 12^{\prime}, 18^{\prime}$ and $30^{\prime}$ from them. Two scans have been made across the plane.

The results on the following four clusters - NGC 2264, NGC 2353, NGC 2362, and NGC 3293 - out of 15 observed are presented below.

NGC 2264: The neutral hydrogen in the region of NGC 2264 and of the stellar association Mon OB1, which contains this cluster, has been investigated by Menon (1956) and later by Raimond (1966) with better frequency and angular resolutions. In this region Raimond has detected three large $\mathrm{HI}$ clouds, two of which, he suggested, are associated with NGC 2264. But the positions of these clouds and their sizes raise doubt as to their immediate connection with the cluster. Most likely these clouds are connected with the association Mon OB1 in general.

The observations at Parkes, made with better angular resolution than that of Raimond, have detected only a small neutral hydrogen cloud in the direction of the cluster (Figure 1). Its angular dimensions are about $30^{\prime}$ and the brightness temperature is almost $20 \mathrm{~K}$. The radial velocity of this cloud (reduced to LSR) is $+10 \mathrm{~km} \mathrm{~s}^{-1}$ and agrees rather well with that of the cluster, which is equal to $+7.5 \mathrm{~km} \mathrm{~s}^{-1}$. Adopting the distance of NGC 2264 to be 760 pc (Raimond, 1966) the linear diameter of the cloud is about $8 \mathrm{pc}$, the density about 10 atoms per $\mathrm{cm}^{3}$ and the mass about $70 M_{\odot}$. Thus the sum of masses of neutral and ionized hydrogen (Tovmasjan, 1967), associated with NGC 2264 is about 80 solar masses.

$N G C$ 2353: The line profiles taken through the position of this cluster have revealed 
no neutral hydrogen cloud structure. All profiles at velocities near the radial velocity of the cluster are sufficiently smooth. Thus there is no noticeable amount of $\mathrm{HI}$ connected with NGC 2353. The attempts to detect $\mathrm{H}_{\mathrm{II}}$ in the vicinity of this cluster were also negative (Tovmasjan, 1967).

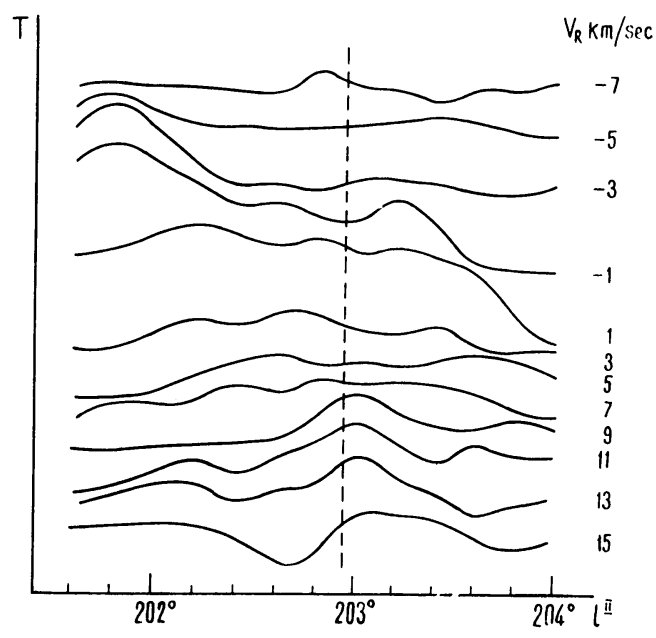

Fig. 1. Line profiles taken through the position of NGC 2264 with the narrow-band receiver.

$N G C$ 2362: This is a small O-type cluster with angular dimensions equal to about 7 '. Line observations have revealed a neutral hydrogen cloud of rather symmetrical form at a distance of about $30^{\prime}$ to the southwest from its centre. The diameter of the cloud is about 25'. The cloud is seen at radial velocities in the range of $30 \mathrm{~km} \mathrm{~s}^{-1}$ while the radial velocity of the cluster is $15 \mathrm{~km} \mathrm{~s}^{-1}$. Both the position and the difference in radial velocities suggest that the cloud is not connected with NGC 2362. It is rather associated with a radio source of comparable size at an adjacent position detected by continuum observations (Tovmasjan, 1967). The position of NGC 2362 and both radio sources (in line emission and continuum) are shown in Figure 2.

Thus no hydrogen is associated with this cluster as well.

NGC 3293: The region of this cluster is very rich with large Hil complexes (Menon, 1956). The hydrogen line observations have also revealed here a complex structure.

The line profiles show the existence of a cloud which is most likely associated with NGC 3293. The radial velocity of the cloud is about $-14 \mathrm{~km} \mathrm{~s}^{-1}$, which is just that of the cluster. The centre of this cloud is projected at the southern edge (in galactic coordinates) of the cluster. The diameter of the cloud is about $20 \mathrm{pc}$ and the brightness temperature is $\sim 24 \mathrm{~K}$. The density in this cloud is $\sim 1.5$ atoms per $\mathrm{cm}^{3}$ and its mass is about $150 M_{\odot}$. These quantities are obtained assuming that the cloud is at the distance of NGC 3293, which is $2.6 \mathrm{kpc}$. If only this neutral hydrogen cloud is associated with NGC 3293 then its total gas content is about $300 M_{\odot}$ since the mass of $\mathrm{H}$ II there is $120 M_{\odot}$ (Tovmasjan, 1967). 
Another feature is probably associated with NGC 3293. This feature may be treated assuming a model of an expanding shell (Figure 3). The radial velocity of the shell is about $+2 \mathrm{~km} \mathrm{~s}^{-1}$ and differs from that of the cluster by $16 \mathrm{~km} \mathrm{~s}^{-1}$, since the latter is $-14 \mathrm{~km} \mathrm{~s}^{-1}$. At the distance of the cluster the outer and the inner radii of the shell are $\sim 50 \mathrm{pc}$ and $\sim 35 \mathrm{pc}$ respectively. With the peak brightness temperature of the shell equal to $\sim 30 \mathrm{~K}$ the mean density of hydrogen atoms in it is about 2 atoms per $\mathrm{cm}^{3}$. The total mass of the shell is about $15000 M_{\odot}$. However, because of the large difference in radial velocities of the cluster and of the shell we can not be sure that the shell is really connected with NGC 3293.

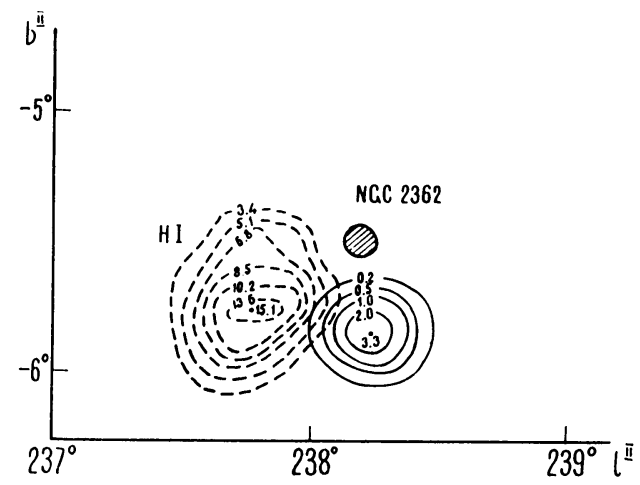

Fig. 2. Map of the region of NGC 2362 with the sources of continuum and line emission. The contours of equal temperature for the neutral hydrogen source are shown for radial velocities of about $+30 \mathrm{~km} \mathrm{~s}^{-1}$.

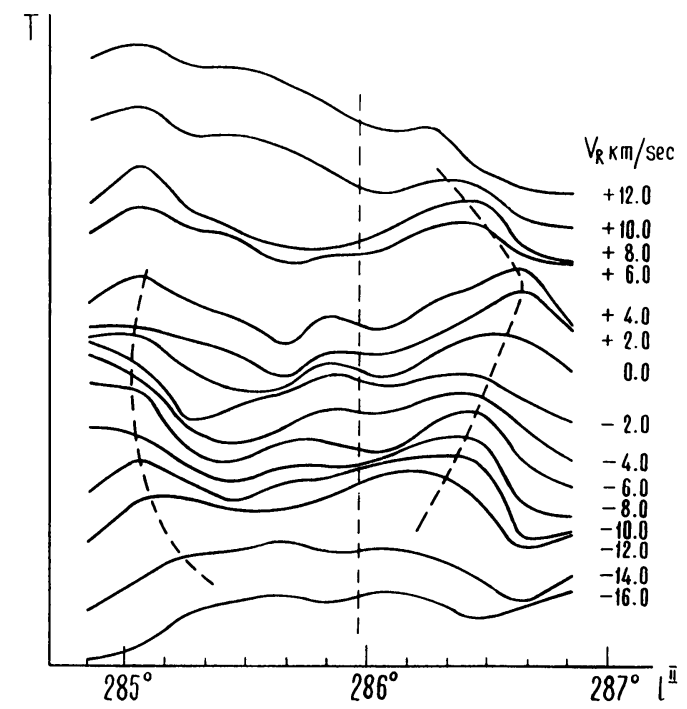

Fig. 3. Line profiles through the position of NGC 3293 obtained with the narrow-band receiver. 
The radial velocity of the small cloud in NGC 2264 is $\sim 7 \mathrm{~km} \mathrm{~s}^{-1}$. The radial velocities for the NGC 3293 are reduced to the Sun.

\section{Acknowledgements}

I am grateful to Drs. E. G. Bowen and J. G. Bolton for permission to use the facilities of the Australian National Radio Astronomical Observatory. My thanks are due to Mrs Liesel Scholem, who has reduced the profiles on the Sydney CDC-3200 computer of the SCIRO Computing Research Section, and Messrs M. Shahoyan and E. Shahbazian, who have helped me in the reduction and analysis of the observations.

\section{References}

Menon, T. K.: 1956, Astron. J. 61, 9.

Raimond, E.: 1966, Bull. Astron. Inst. Netherl. 18, 191.

Tovmasjan, H. M.: 1967, IAU Symposium No. 31, p. 37. 